\title{
Stage IIIB Small Intestinal Adenocarcinoma AJCC v8
}

National Cancer Institute

\section{Source}

National Cancer Institute. Stage IIIB Small Intestinal Adenocarcinoma A/CC v8. NCI Thesaurus. Code C133901.

Stage IIIB includes: Any T, N2, M0. N2: Metastasis in three or more regional lymph nodes. M0: No distant metastasis. (AJCC 8th ed.) 